\title{
Early development of perceptual expertise: Within-basic-level categorization experience facilitates the formation of subordinate-level category representations in 6- to 7-month-old infants
}

\author{
Paul C. QuinN \\ University of Delaware, Newark, Delaware \\ AND \\ JAMES W. TANAKA \\ University of Victoria, Victoria, British Columbia, Canada
}

\begin{abstract}
A behavioral manifestation of perceptual expertise is the shift in recognition downward toward the subordinate level. Here, a familiarization-novelty preference procedure was used to determine whether 6- to 7-monthold infants could be induced to form category representations for cats and dogs at the subordinate level. In Experiment 1, the infants succeeded in forming subordinate-level category representations for beagle dogs and Siamese cats, but only when provided with previous experience on a subordinate-level category-formation task from within the same basic-level category (i.e., Saint Bernard dogs or tabby cats). When the prior categoryformation task involved a different basic-level category, subsequent subordinate-level category learning was not in evidence. The preferences in Experiment 1 were shown in Experiments 2 and 3 not to be attributable to a priori preference or within-category discrimination failure. The findings suggest that within-basic-level categorization experience can facilitate the formation of subordinate-level category representations in the first half-year of life.
\end{abstract}

There has been a recent investigative effort aimed at understanding the changes in categorization, identification, and recognition of objects that occur with the acquisition of perceptual expertise in adults (e.g., Palmeri, Wong, \& Gauthier, 2004). A behavioral manifestation of perceptual expertise in adults is the "downward shift" in recognition, in which experts recognize objects within their domain of expertise at a subordinate rather than a basic level of categorization (Tanaka, 2001; Tanaka \& Taylor, 1991). For example, a bird expert is likely to identify a sparrow as a "sparrow" rather than as a "bird." A recent study conducted with adult participants suggested that training consisting of perceptual categorization at the subordinate level facilitated the learning of novel subordinate-level categories from within the same basic-level category (Tanaka, Curran, \& Sheinberg, 2005). In particular, laboratory training of wading bird expertise transferred to other species of wading birds, but not to owls (and vice versa). The results imply that experience with categorization of objects from within the same basic level as the target category may tune one's perceptual system to features that are diagnostic for subordinate-level category contrasts within that basic-level category.
The study of perceptual expertise in adults has led developmentalists to ask how early and on what bases perceptual expertise might arise during the course of our initial interactions with the environment (Gauthier \& Nelson, 2001). Investigating whether expertise can emerge in young infants, a participant population with limited realworld experience, provides a way of assessing the contributions of specific learning experiences to acquired expertise. To this end, Quinn (2005) has reported differences in the ways that young infants, 3-4 months of age, represent categories of humans and nonhuman animals, leading to the argument that young infants' considerable familiarity with humans may generate the first domain of perceptual expertise. For example, the category representation for humans (presented as whole-bodied images) has been shown to consist of subordinate-like representations of individual exemplars, whereas the category representation of nonhuman animal species was found to be based on summary information on the order of a prototype that represents an averaging of the familiar exemplars (Quinn \& Eimas, 1998). The possibility that humans may be represented at a more advanced stage of processing than nonhuman animals is

P. C. Quinn, pquinn@udel.edu 
consistent with a hypothesized representational shift from a summary-based structure to exemplar-based memory during the time course of category learning in adults (Gauthier \& Tarr, 1997; Johansen \& Palmeri, 2002; Smith \& Minda, 1998). In addition, the category representation of humans by infants appears to be based on the overall structure of the stimuli- for example, a head region adjoining an elongated body axis with skeletal appendages (Quinn, 2004). The finding that the human representation is based on global information contrasts with results showing that representations for nonhuman animal species are based on part or attribute (i.e., featural) information-heads, in the case of cats versus dogs (Quinn, Eimas, \& Tarr, 2001). These data on how infants represent humans correspond with the finding that expert object recognition by adults is characterized by holistic-configural processing (Gauthier \& Tarr, 2002; but see Robbins \& McKone, 2007).

Although the argument that young infants are perceptual experts on humans is made on the basis of experiences occurring outside the laboratory - in this case, repeated encounters with conspecifics (Kelly et al., 2005, 2007; Quinn, Yahr, Kuhn, Slater, \& Pascalis, 2002) - the Tanaka et al. (2005) findings with adults raise questions about whether laboratory training can induce perceptual expertise early in ontogenesis. Developmental evidence on the acquisition of perceptual categories that are presumably formed during online learning occurring within an experimental task is consistent with the idea that the subordinate level of categorization is the last to be acquired during early development (Quinn, 2002). For example, Quinn and Johnson (2000) reported that 2-month-old infants could form a global (superordinate) category representation for mammals that included instances of novel mammal categories but excluded instances of furniture. However, infants at this age did not form a basic-level category representation for cats that excluded dogs, rabbits, or elephants. Approximately 6 weeks later in development, at 3-4 months, infants were shown to form both a global category representation for mammals that excluded furniture (Behl-Chadha, 1996), as well as basic-level category representations for cats that excluded birds, dogs, horses, and tigers, and for horses that excluded cats, giraffes, and zebras (Eimas \& Quinn, 1994). The global-to-basic developmental trend in infant perceptual category learning is noteworthy because it contrasts with the traditional basicto-superordinate account of category learning in children (Rosch, Mervis, Gray, Johnson, \& Boyes-Braem, 1976).

The general-to-specific view predicts that subordinatelevel categories should be the last level of categorization to be acquired. In accord with this view, it has been shown that 3- to 4-month-olds were not able to form subordinatelevel category representations that distinguished Siamese from tabby cats or Saint Bernard from beagle dogs (Quinn, 2004). Older infants, 6-7 months of age, formed partial category representations for tabby cats that excluded Siamese cats and for Saint Bernard dogs that excluded beagles. However, the subordinate category representations were not complete, because the Siamese category failed to exclude tabby and the beagle category did not exclude Saint Bernard. The foregoing results suggest that category learning begins first at a general, superordinate category level, proceeds to an intermediate, basic level, and is followed by the acquisition of specific, subordinate-level categories. Although subordinate-level learning has begun by 6-7 months of age, it is not fully developed to the degree that the infant's subordinate-level category contrasts do not mutually exclude each other.

\section{EXPERIMENT 1}

In the present study, given the Tanaka et al. (2005) findings with adults, we inquired into whether initial participation in a subordinate-level category-formation task would facilitate the subsequent learning of different subordinate-level category representations from within the same basic-level category in 6- to 7-month-old infants. Specifically, following from the results of Quinn (2004), we investigated whether the infants could be induced to form subordinate-level category representations for beagles (or Siamese cats) if they were initially allowed to form a subordinate-level category representation for Saint Bernards (or tabbies). In contrast to the conditions in Quinn (2004) that resulted in null outcomes, we sought to determine whether prior experience with a subordinatelevel category contrast from the same basic-level category would bring about successful subordinate-level category formation. Whereas the infants in Quinn (2004) were provided with only a single categorization task presenting a subordinate-level contrast, the infants in the present study were provided with two subordinate-level categorization tasks from the same basic-level class presented in succession. To head off the potential criticism that infants might respond more successfully on any category-learning task that was presented following participation in an initial category-learning task, control conditions were included in which infants were tested for their ability to form category representations for beagles (or Siamese cats) when first presented with tabbies (or Saint Bernards).

On the basis of the findings reported in Quinn (2004), the expectation was that infants in the initial category-learning task would succeed in forming a category representation for Saint Bernards that excludes beagles and for tabbies that excludes Siamese cats. In addition, in the subsequent categorylearning task, if infants could acquire perceptual expertise through laboratory experience in a manner similar to that observed for adults (Tanaka et al., 2005), the prediction was that performance would be above-chance in the same basiclevel group (cat $\rightarrow$ cat or dog $\rightarrow$ dog), but at chance for the different basic-level group (cat $\rightarrow \operatorname{dog}$ or $\operatorname{dog} \rightarrow$ cat). That is, infants should succeed in forming a category representation for beagles that excludes Saint Bernards and for Siamese cats that excludes tabbies (something they did not do when only a single subordinate-level category-learning task was administered in Quinn [2004]), but only when they had previously experienced a subordinate-level categorylearning task from the same basic-level category.

\section{Method}

Participants. The participants were sixty-four 6- to 7-monthold infants (32 females) with a mean age of 195.62 days ( $S D=$ 
Table 1

Design of Experiment 1

\begin{tabular}{|c|c|c|c|c|}
\hline \multirow[b]{2}{*}{ Condition } & \multicolumn{2}{|r|}{ Part 1} & \multicolumn{2}{|r|}{ Part 2} \\
\hline & Familiarization & Test & Familiarization & Test \\
\hline \multicolumn{5}{|l|}{ Same basic level } \\
\hline Either: & 12 tabbies & Novel tabby vs. novel Siamese & 12 Siamese & Novel Siamese vs. novel tabby \\
\hline Or: & 12 Saint Bernards & Novel Saint Bernard vs. novel beagle & 12 beagles & Novel beagle vs. novel Saint Bernard \\
\hline \multicolumn{5}{|c|}{ Different basic level } \\
\hline Either: & 12 tabbies & Novel tabby vs. novel Siamese & 12 beagles & Novel beagle vs. novel Saint Bernard \\
\hline Or: & 12 Saint Bernards & Novel Saint Bernard vs. novel beagle & 12 Siamese & Novel Siamese vs. novel tabby \\
\hline
\end{tabular}

11.46 days). We tested 12 additional infants, but 7 failed to complete the procedure due to fussiness, and 5 were excluded from the data analysis because of failure to compare the stimuli during one or both preference tests.

Stimuli. The stimuli were the same as those used in Quinn (2004). They consisted of 18 colored photographs from each of the four categories. The pictures were cut from a variety of pet books and animal field guides depicting the animals in their natural habitats. Pictures in all of the categories were chosen to represent a variety of stances and orientations. Each picture contained a single animal that had been cut away from its background, centered, and mounted onto a white poster board for presentation to the infants. The pictures were selected to be of approximately the same size. As a result, the infants were required to use cues such as shape, parts, or the pattern of correlation across the parts - cues that have been shown to be diagnostic of category membership for both infants and adults (Quinn et al., 2001; Rosch et al., 1976; Younger, 1985).

Apparatus. All of the infants were tested in a visual preference apparatus, modeled after the one described by Fagan (1970). The apparatus had a display panel, onto which were attached two compartments to hold the poster-board stimuli. The display panel was situated approximately $30.5 \mathrm{~cm}$ in front of the infant. A peephole located midway between the two display compartments permitted an observer to record the infant's visual fixations. A second peephole located directly below the first peephole permitted a pinhole camera and a video recorder to record infants' gaze duration.

Procedure. All of the infants were brought to the laboratory by a parent and were seated in a reclining position on the parent's lap. There were two experimenters, both of whom were naive to the hypotheses under investigation. The first experimenter positioned the apparatus so that the midline of the infant's head was aligned with the midline of the display panel. When the display panel was open, the infant could see the experimenter from the midsection up, in addition to a portion of the room that was a light background color. The experimenter selected the appropriate stimuli and loaded them into the compartments of the display panel. The experimenter then elicited the infant's attention and closed the panel, thereby exposing the stimuli to the infant. The parent was unable to see the stimuli.

During each trial, the first experimenter observed the infant through the peephole and recorded visual fixations to the left and right stimuli by means of two electronic stopwatches, one of which was held in each hand. Between trials, the experimenter opened the panel, changed the stimuli, recorded infant looking times, reobtained the infant's attention, recentered his or her gaze, and closed the panel. The intertrial interval was approximately $7.5 \mathrm{sec}$. When estimates of spontaneous preference were obtained in Experiment 2, the second experimenter did not participate other than to time the trials and signal when a trial was to end. In experiments that measured the categorization or discrimination of the stimuli (Experiments 1 and 3), the first and second experimenters changed places for the test trials. The experimenter who had presented stimuli and measured infant fixations during familiarization now measured trial duration and signaled the end of each test trial, whereas the second experimenter presented the test stimuli and measured infant fixations. The second experimenter was always naive with respect to the familiar category (Experiment 1) or familiar stimulus (Experiment 3). The two experimenters changed roles across infants.

Interobserver agreement - as determined by comparing the looking times measured by the experimenter using the center peephole and those obtained by an additional naive observer measuring looking times offline from videotape records - was calculated for the preference test trials of 16 randomly selected infants. Average level of agreement for novel category preference scores was $97.85 \%$ $(S D=2.42 \%)$.

The infants were randomly assigned to each of two experimental groups, defined by whether the initial category-learning task came from the same basic-level category or a different basic-level category as the category to be tested subsequently. Specifically, as shown in Table 1, infants in the same basic-level group were initially presented with 12 tabbies (or Saint Bernards) and then given a preference test between a novel tabby and a novel Siamese cat (or a novel Saint Bernard and a novel beagle). They were subsequently presented with 12 Siamese cats (or beagles) and then given a preference test between a novel Siamese cat and a novel tabby (or a novel beagle and a novel Saint Bernard). Infants in the different basic-level group were provided with the same initial category-learning experience, but then subsequently presented with 12 beagles (or Siamese cats) and given a preference test between a novel beagle and a novel Saint Bernard (or a novel Siamese cat and a novel tabby).

For both the same and different basic-level groups, each of the familiarization periods consisted of six 15 -sec familiarization trials. On each trial, two different exemplars of the category were presented. Each of the preference tests consisted of two 10-sec trials. The left-right positioning of the novel- and familiar-category stimuli was counterbalanced across infants on the first test trial and reversed on the second test trial. All of the familiarization and test stimuli were randomly selected for each infant. For a given infant, the random selection was accomplished without replacement. Because the stimuli were selected from an available pool of 18 stimuli for each category, this procedure made it possible for each infant to receive only a single presentation of each exemplar. Thus, for example, even in the same basic-level condition, the stimuli in Part 2 would not have been observed in Part 1.

\section{Results and Discussion}

Part I: Familiarization trials. Individual looking times were summed over both stimuli on each trial and then averaged across the first three trials and the last three trials. The mean looking times are shown in Table 2 for the same basic-level (cat $\rightarrow$ cat, dog $\rightarrow$ dog, combined) and different basic-level conditions (cat $\rightarrow$ dog, dog $\rightarrow$ cat, combined). An experimental condition (same basic level vs. different basic level) $\times$ familiar category (cat vs. dog) $\times$ trial blocks (1-3 vs. 4-6) ANOVA performed on the individual scores revealed only a marginally reliable effect of familiar category $[F(1,60)=3.19, p=.075]$. No other effects were significant $(F<0.62$, in each instance). 
Table 2

Mean Fixation Times (in Seconds) and Standard Deviations During the Familiarization Trials of Experiment 1

\begin{tabular}{|c|c|c|c|c|c|c|c|c|}
\hline \multirow[b]{3}{*}{ Group } & \multicolumn{4}{|c|}{ Part 1} & \multicolumn{4}{|c|}{ Part 2} \\
\hline & \multicolumn{2}{|c|}{ Trials $1-3$} & \multicolumn{2}{|c|}{ Trials 4-6 } & \multicolumn{2}{|c|}{ Trials $1-3$} & \multicolumn{2}{|c|}{ Trials 4-6 } \\
\hline & $M$ & $S D$ & $M$ & $S D$ & $M$ & $S D$ & $M$ & $S D$ \\
\hline \multicolumn{9}{|c|}{ Same Basic Level } \\
\hline Cat $\rightarrow$ cat & 7.34 & 3.37 & 6.63 & 3.83 & 6.54 & 1.65 & 6.47 & 3.33 \\
\hline $\operatorname{Dog} \rightarrow \operatorname{dog}$ & 5.53 & 2.19 & 5.41 & 1.58 & 5.90 & 2.58 & 5.74 & 2.90 \\
\hline Combined & 6.44 & 2.94 & 6.02 & 2.95 & 6.22 & 2.16 & 6.10 & 3.09 \\
\hline \multicolumn{9}{|c|}{ Different Basic Level } \\
\hline Cat $\rightarrow$ dog & 6.89 & 3.33 & 6.74 & 2.88 & 6.61 & 3.29 & 7.07 & 2.96 \\
\hline Dog $\rightarrow$ cat & 5.93 & 2.35 & 6.03 & 2.57 & 6.13 & 2.38 & 6.51 & 2.37 \\
\hline Combined & 6.41 & 2.88 & 6.38 & 2.71 & 6.37 & 2.83 & 6.79 & 2.65 \\
\hline
\end{tabular}

The marginally significant effect of familiar category indicates that the infants looked somewhat longer at the cat than at the dog stimuli, a finding that is consistent with what was observed in Quinn (2004). The fact that the infants did not show a reliable decrement in looking time during familiarization is consistent with how infants in the same age group responded to the presentation of subordinate-level categories of cats and dogs in Quinn (2004), and is also in accord with how infants responded to global (superordinate) and basic-level categoryfamiliarization experiences in Behl-Chadha (1996) and Eimas and Quinn (1994). In each of these instances, it is likely that the presentation of varied and discriminably different exemplars was sufficient to maintain infant attention throughout the familiarization period. One further note is that one would not have expected an effect of experimental condition, given that infants in both conditions were treated equivalently in terms of being presented with cats or dogs.

Part I: Preference test trials. Each infant's looking time to the stimulus from the novel category was divided by the total looking time to both test stimuli and converted to a percentage score. The mean preference scores to the novel category are shown in Table 3 for the same basiclevel (cat $\rightarrow$ cat, $\operatorname{dog} \rightarrow$ dog, combined) and different basic-level (cat $\rightarrow \operatorname{dog}$, dog $\rightarrow$ cat, combined) conditions. For the same basic-level condition, the preference scores for all of the groups were found to be reliably above the chance preference of $50 \%$ [cat $\rightarrow$ cat, $M=61.45, S D=$ $16.90, t(15)=2.71, p<.02 ; \operatorname{dog} \rightarrow \operatorname{dog}, M=62.90$, $S D=18.50, t(15)=2.79, p<.02 ;$ combined $M=62.17$, $S D=17.45, t(31)=3.94, p<.001]$. The same was true for the preference scores of the different basic-level condition [cat $\rightarrow \operatorname{dog}, M=57.40, S D=13.88, t(15)=2.13$, $p<.05 ; \operatorname{dog} \rightarrow$ cat $, M=64.27, S D=17.24, t(15)=3.31$, $p<.01$; combined $M=60.84, S D=15.79, t(31)=3.88$, $p<.001]$. The results replicate those reported in Quinn (2004): Infants formed subordinate-level category representations for Saint Bernards that excluded beagles, and for tabbies that excluded Siamese cats. In addition, planned comparisons revealed that the combined novel category preference observed for the different basic-level group did not differ from the combined novel category preference observed for the same basic-level group $[t(62)=0.32$, $p>.20]$. Once more, this result was to be expected, given that the two groups were exposed to identical subordinatelevel category contrasts.

Part 2: Familiarization trials. Mean looking times are shown in Table 2 . An experimental group $\times$ familiar category $\times$ trial block ANOVA was performed on the individual scores and did not uncover any significant effects ( $F<1.02$, in each instance). Although the looking time to the cats $(M=6.41, S D=2.15)$ was nominally higher than that to the $\operatorname{dogs}(M=6.33, S D=2.57)$, in this case, the effect of familiar category was not reliable. In addition, as was true for the initial familiarization period, the variation among the exemplars likely maintained infant attention throughout the trials.

Part 2: Preference test trials. The mean preference scores to the novel category are shown in Table 3 . For infants in the same basic-level category condition, the preference scores were again all reliably above chance [cat $\rightarrow$ cat, $M=64.91, S D=13.47, t(15)=4.43, p<.001 ; \operatorname{dog}$ $\rightarrow \operatorname{dog}, M=60.73, S D=11.55, t(15)=3.72, p<.01$; combined $M=62.82, S D=12.52, t(31)=5.79, p<$ $.001]$. By contrast, for infants in the different basic-level condition, none of the preference scores reached significance against chance [cat $\rightarrow \operatorname{dog}, M=50.11, S D=17.71$, $t(15)=0.02, p>.20 ; \operatorname{dog} \rightarrow$ cat $, M=56.68, S D=12.96$, $t(15)=2.06, p>.05$; combined $M=53.40, S D=15.62$, $t(31)=1.23, p>.20]$. In addition, planned comparisons confirmed that the combined novel category preference observed for the same basic-level group differed reliably from the combined novel category preference observed for the different basic-level group $[t(62)=2.66, p<.01]$.

Table 3

Mean Preference Scores (in Percentages) for the Novel Category During the Test Trials of Experiment 1

\begin{tabular}{ccccc}
\hline & \multicolumn{4}{c}{ Preference } \\
\cline { 2 - 3 } \cline { 5 - 5 } Group & \multicolumn{2}{c}{ Part 1 } & & \multicolumn{2}{c}{ Part 2 } \\
\cline { 2 - 3 } \cline { 5 - 5 } & $M$ & $S D$ & & $S D$ \\
\hline Same Basic Level & 61.45 & 16.90 & 64.91 & 13.47 \\
Cat $\rightarrow$ cat & 62.90 & 18.50 & 60.73 & 11.55 \\
Dog $\rightarrow$ dog & 62.17 & 17.45 & 62.82 & 12.52 \\
Combined & & & & \\
Different Basic Level & 57.40 & 13.88 & 50.11 & 17.71 \\
Cat $\rightarrow$ dog & 64.27 & 17.24 & 56.68 & 12.96 \\
Dog $\rightarrow$ cat & 60.84 & 15.79 & 53.40 & 15.62 \\
Combined & & & &
\end{tabular}


The overall pattern of results indicates that the infants were able to form a subordinate-level category representation for Siamese cats that excluded tabbies and for beagles that excluded Saint Bernards, but only when they had been presented with a prior subordinate-level category-formation task in which the initial subordinate-level category was from the same basic-level category. This pattern of outcomes will become even more apparent in Experiment 2 when the novel category preference scores are compared with spontaneous preference scores measuring baseline preferences for one category versus another.

\section{EXPERIMENT 2}

The results of Experiment 1 support the view that initial subordinate-level category experience can enhance the infant's acquisition of a new subordinate-level category representation-provided that both subordinate-level categories are from the same basic-level category. These findings stand in contrast to the previous results reported by Quinn (2004), in which the same subordinate-level category representations were not acquired when a single subordinate category was presented in isolation. However, one can argue that the same performance criterion that was applied to the infants reported in Quinn (2004) was not applied to the infants reported in Experiment 1. That is, in the present study, the conclusions have thus far been drawn from comparison of infant novel category preference after familiarization with chance performance, whereas in Quinn (2004), novel category preferences were compared using groups of infants who were tested for a priori preferences. The Quinn (2004) analysis follows from the rationale that if a preference for a novel category is observed and is to be attributed to a process of categorization, it must be demonstrated that the novel category preference was not a consequence of an a priori preference. In order to apply this same performance criterion in the present study, we conducted Experiment 2 to measure spontaneous preference for one class of stimuli over another before familiarization with one or the other class. Six- to seven-month-old infants were presented with different, randomly selected pairs of pictures from two different categories on each trial (Siamese cat vs. tabby, beagle vs. Saint Bernard). The presence of spontaneous perceptual preferences was estimated from the pattern of looking across eight 10 -sec trials. The number of trials in the spontaneous preference task (i.e., 8) was equivalent to the number of trials presented to infants in the categorization task of Experiment 1 and in Quinn (2004) (i.e., 6 familiarization +2 test). In addition, the duration of the trials in the spontaneous preference task (i.e., $10 \mathrm{sec}$ ) was equivalent to the duration of the test trials in the categorization task of Experiment 1 and in Quinn (2004) - that is to say, those trials that contrasted instances of the two categories.

\section{Method}

Participants. The participants were thirty-two 6- to 7-month-old infants (18 females) with a mean age of 189.00 days $(S D=8.91$ days). Two additional infants were tested but did not complete the procedure because of fussiness.
Stimuli and Apparatus. The stimuli and apparatus were the same as those used in Experiment 1.

Procedure. Sixteen infants were randomly assigned to each of two preference conditions: the pairings of Siamese cat versus tabby, and beagle versus Saint Bernard. On each of eight 10 -sec trials, infants were presented with an instance of one category paired with an exemplar from another category. Different pairings were randomly chosen for each infant on each trial. The left-right positioning of the two categories was counterbalanced across infants on the first test trial and reversed on each successive trial. Interobserver agreement was calculated for the spontaneous perceptual preference scores of 8 randomly selected infants, and averaged $97.49 \%(S D=4.37)$.

\section{Results and Discussion}

Spontaneous perceptual preference scores were calculated for tabby, Siamese, Saint Bernard, and beagle when exemplars of these categories were paired with Siamese, tabby, beagle, and Saint Bernard, respectively. The preference score was determined for each infant by dividing the summed looking time to one category of stimuli over all eight trials by the summed looking time to both categories over the eight trials. This score was then converted to a percentage and averaged across infants to yield a mean spontaneous preference score for each test. The mean spontaneous preference scores are shown in Table 4. $t$ tests (chance preference of 50\%) revealed no significant preference for tabby over Siamese $[t(15)=1.27, p>.20]$ or Saint Bernard over beagle $[t(15)=-0.01, p>.20]$.

On the basis of the spontaneous perceptual preference scores, the novel category preference scores observed for the subgroups in Experiment 1 can now be subjected to the same analysis reported in Quinn (2004). In particular, the novel category preference scores from Experiment 1 can be compared with the spontaneous preference scores from Experiment 2. Consider first the results from Part 1 of Experiment 1 . In the same and different basic-level conditions from Experiment 1, infants showed mean novel category preferences for Siamese cats, after familiarization with tabby cats, of $61.45 \%(S D=16.90 \%)$ and $57.40 \%$ $(S D=13.88 \%)$, values that were reliably different from the spontaneous preference for Siamese cats when paired with tabby cats $[t(30)>2.47, p<.02$, in both cases]. Also, in the same and different basic-level conditions from Part 1 of Experiment 1, infants showed mean novel category preferences for beagles, after familiarization with Saint Bernards, of $62.90 \%(S D=18.50 \%)$ and $64.27 \%$ $(S D=17.24 \%)$, scores that were significantly different from the spontaneous preference for beagle when it was paired with Saint Bernard $[t(30)>2.66, p<.02$, in both cases]. These results provide empirical confirmation of the results reported in Quinn (2004) and substantiate the claims made on the basis of the Part 1 data from Experi-

Table 4

Mean Spontaneous Perceptual Preference Scores (in Percentages) in Experiment 2

\begin{tabular}{lrr}
\hline \multicolumn{1}{c}{ Preference For } & \multicolumn{1}{c}{$M$} & \multicolumn{1}{c}{$S D$} \\
\hline Tabby (when paired with Siamese) & 52.68 & 8.47 \\
Siamese (when paired with tabby) & 47.32 & 8.47 \\
Saint Bernard (when paired with beagle) & 49.97 & 11.06 \\
Beagle (when paired with Saint Bernard) & 50.03 & 11.06
\end{tabular}


ment 1 -namely, that infants can form a category representation for tabby cats that excludes instances of Siamese cats, and that infants can form a category representation for Saint Bernards that excludes instances of beagles.

With regard to the Part 2 results from Experiment 1, infants in the same and different basic-level conditions showed mean novel category preferences for tabby cats, after familiarization with Siamese cats, of $64.91 \%$ ( $S D=$ $13.47 \%)$ and $56.68 \%(S D=12.96 \%)$. When these values were compared with the spontaneous preference for a tabby cat when paired with a Siamese cat, the difference was significant in the same basic-level condition $[t(30)=$ $3.07, p<.01]$, but not in the different basic-level condition $[t(30)=1.03, p>.20]$. In addition, infants in the same and different basic-level conditions from Part 2 of Experiment 1 showed mean novel category preferences for Saint Bernards, after familiarization with beagles, of $60.73 \%(S D=11.55 \%)$ and $50.11 \%(S D=17.71 \%)$. When these scores were compared with the spontaneous preference for Saint Bernards, when paired with beagles, the difference was reliable in the same basic-level condition $[t(30)=2.69, p<.02]$, but not in the different basic-level condition $[t(30)=0.03, p>.20]$. The findings from the Part 2 analyses provide further support for the conclusions reached in Experiment 1 on the basis of the comparisons of the mean novel category preferences with chance. Specifically, infants in Part 2 were found to form a category representation for Siamese cats that excluded instances of tabbies and a category representation for beagles that excluded exemplars of Saint Bernards, but only when these tasks were preceded by a different subordinate-level categorization task chosen from within the same basic-level category in Part 1.

Although the findings from Experiment 2 have upheld the conclusions from Experiment 1, there is still one more issue that must be examined before categorization can be considered to be decisively documented. In particular, given that categorization is only in evidence when one has demonstrated equivalent responding to discriminably different instances from a common class, it must be shown that the individual exemplars of the categories under investigation are discriminably different. Instances from within the tabby and Saint Bernard categories were shown to be differentiable by 6- to 7-month-olds in Quinn (2004). The question of whether exemplars from within the Siamese cat and beagle categories can be distinguished was examined in Experiment 3.

\section{EXPERIMENT 3}

We conducted Experiment 3 to determine whether the individual exemplars of the Siamese cat and beagle categories could be differentiated from one another. If within-category differences between exemplars were not detectable by the infants, then the categorization task of Experiment 1 would have been reduced to a betweencategory discrimination task, one in which infants detected the difference between a single instance from the familiar category (given that the differences among instances within the familiar category were undetectable) and an exemplar from the novel category. The procedure used to measure discrimination of instances within the categories involved presenting a randomly selected member of the Siamese cat or beagle categories for a single 15 -sec trial. A single trial was employed because it was in accord with the duration of presentation of each exemplar during the familiarization portion of the categorization task in Experiment 1. Immediately following familiarization, two 10 -sec preference test trials were administered, in which the familiar stimulus was paired with a novel instance from the same category, also randomly selected.

\section{Method}

Participants. The participants were thirty-two 6- to 7-monthold infants (14 females) with a mean age of 196.31 days $(S D=$ 12.46 days). One additional infant was tested but did not complete the procedure due to fussiness.

Stimuli. The stimuli were the Siamese cat and beagle exemplars used in the previous experiments.

Procedure. Sixteen infants were randomly assigned to each of two experimental groups: Siamese cat and beagle. Each infant received a single 15 -sec familiarization trial, during which a single Siamese cat or beagle, randomly selected for each infant, was presented in both compartments of the display stage. Immediately following familiarization, each infant was presented with two 10-sec test trials pairing the familiar Siamese cat with a novel Siamese cat or the familiar beagle with a novel beagle. The novel Siamese cat or beagle was also randomly selected for each infant. The left-right positioning of the novel stimulus was counterbalanced across infants on the first trial and reversed on the second trial. Interobserver agreement was calculated for the novelty preference scores of 8 randomly selected infants, and averaged $97.29 \%(S D=2.04 \%)$.

\section{Results and Discussion}

Familiarization trial. Individual looking times were summed over the left and right copies of the stimulus on the familiarization trial and then averaged across infants. Mean looking times to the exemplars of the Siamese cat and beagle are presented in Table 5. The difference in the mean looking times was not significant $[t(30)=0.21, p>$ $.20]$.

Preference test trials. Each infant's looking time to the novel stimulus was divided by the looking time to both test stimuli and then converted to a percentage score. The mean preference scores for the novel stimulus for the two familiarization conditions are shown in Table 5. Both means were reliably above chance $[t(15)=2.14$ and 2.58 for the Siamese cat and beagle conditions, respectively, $p<.05$, in both cases]. Infants familiarized with either a Siamese cat or a beagle preferred a novel exemplar from the familiar category over the familiar exemplar from the familiar category. These data indicate that 6 - to

Table 5

Mean Fixation Times (in Seconds) During the Familiarization Trial and Mean Novelty Preference Scores (in Percentages) During the Preference Test Trials in Experiment 3

\begin{tabular}{|c|c|c|c|c|}
\hline \multirow{2}{*}{$\begin{array}{l}\text { Familiar } \\
\text { Stimulus }\end{array}$} & \multicolumn{2}{|c|}{ Fixation Time } & \multicolumn{2}{|c|}{$\begin{array}{c}\text { Novelty } \\
\text { Preference }\end{array}$} \\
\hline & $M$ & $S D$ & $M$ & $S D$ \\
\hline Siamese & 8.50 & 3.50 & 57.44 & 13.88 \\
\hline Beagle & 8.26 & 2.85 & 59.07 & 14.08 \\
\hline
\end{tabular}


7-month-olds can discriminate instances from within the Siamese cat and beagle categories. The findings uphold the conclusion from Experiments 1 and 2 that infants were able to form category representations of discriminably different instances of Siamese cats and beagles that excluded exemplars of tabby cats and Saint Bernards, respectively.

\section{GENERAL DISCUSSION}

The results of Experiment 1 demonstrated that infants first participating in one subordinate-level categoryformation task (e.g., with Saint Bernards) were able to subsequently learn a second subordinate-level category from the same basic-level category (e.g., beagles), but not from a different basic-level category (e.g., Siamese cats). It is important to emphasize that the second subordinatelevel category representation that was learned was not learned when presented in isolation in Quinn (2004). In addition, the outcome of the different basic-level condition shows that infants will not simply learn a second category representation more effectively when provided with an opportunity to form a first category representation. Moreover, Experiments 2 and 3 established that the novel category preferences observed in Experiment 1 could not be attributed to a priori preferences or within-category discrimination failure.

The novel category preferences of the infants in the same basic-level group provide support for the argument that infant categorization performance is flexible (French, Mareschal, Mermillod, \& Quinn, 2004). The infants in the same basic-level conditions of Experiment 1 showed a novel category preference for Siamese cats (or beagles) in Part 1, and then displayed a novel category preference for tabbies (or Saint Bernards) in Part 2. It is noteworthy that the novel category preference in Part 2 was just as strong as that in Part 1, even though the infants had experienced the novel subordinate category before as the familiar category.

It is interesting to consider the factors that might account for the subordinate-level categorization performance that was observed in the Part 2 preference test trials of Experiment 1. In contrast to the Quinn (2004) study, infants in the same basic-level category condition received additional exposure to subordinate-level exemplars during the Part 2 familiarization trials. Thus, from a pure exposure standpoint, the same basic-level group had an advantage relative to the different basic-level group, although it should be emphasized once more that the additional perceptual experience facilitated categorization only when it came from within the same basic-level category.

One possibility that should be considered in terms of accounting for the subordinate-level categorization results in Part 2 of Experiment 1 is that infants in the two conditions may have attended to different features. In the different basic-level conditions, in which infants go from cat (or dog) in Part 1 to dog (or cat) in Part 2, the features extracted in Part 1 did not seem to be useful in Part 2, at least to the extent that they were in the same basiclevel conditions in which infants go from cat (or dog) in Part 1 to cat (or dog) in Part 2. It may be the case that the different basic-level condition (cat + dog) leads infants to attend to features common to both cats and dogs, whereas the same basic-level conditions (cat to cat and dog to dog) better underscore the features that are relevant within each category. Although this suggestion would need to be confirmed in experimental work with artificial categories, in which the stimuli could be manipulated to include common or distinctive features, it is consistent with previous evidence showing that infants form more inclusive or exclusive category representations depending on the variability of the exemplars shown during familiarization (Oakes, Coppage, \& Dingel, 1997; Quinn, Eimas, \& Rosenkrantz, 1993; Younger \& Fearing, 1999). Given Tanaka et al.'s (2005) report that in adults, laboratorytrained owl expertise transferred to other species of owls, but not to wading birds (and vice versa), the combined results suggest that stimulus variability in learning is crucial to new category acquisition and generalization in both infants and experts.

The reported findings are also in accord with an account of object recognition and categorization that emphasizes flexible feature creation (Schyns, Goldstone, \& Thibaut, 1998). This model maintains that the features that come to represent objects are developed during the task of concept learning. The concepts possessed by an individual at a given moment in developmental time will influence how that individual recognizes subsequently presented objects. This argument has received support from infant work showing that how infants respond to complex displays of visual pattern information is influenced by immediately preceding category-learning experiences (Quinn \& Schyns, 2003; Quinn, Schyns, \& Goldstone, 2006). In particular, infants initially given a category-learning experience with a set of complex polygons marked by an invariant Pac-Man shape later recognized the Pac-Man shape as familiar during subsequent presentation of a set of polygons containing an additional shape that could be alternatively interpreted as consisting of either a circle shape or a Pac-Man shape. Infants who did not receive the prior category-learning experience with the Pac-Man shape represented the circle among the subsequently presented circle-Pac-Man shapes. These findings match well with those presented here: Infants presented initially with a subordinate-level distinction among cat (or dog) categories may have become tuned to features that allowed for separation of the later presented contrast between categories of cats (or dogs). Such tuning was not present when the infants were shown the second category contrast without an initial subordinate-category-learning experience (Quinn, 2004) or when the initial subordinate-categorylearning experience was from a different basic-level category and likely tuned infants into a different feature cluster as being relevant to categorization. The results of both the previous Pac-Man-circle studies and the present cat-dog investigations suggest that initial category learning serves to tune perceptual systems to features that will be useful to learning subsequently presented categories.

The theme of perceptual tuning via category learning has also been examined by investigators studying the neural basis of object recognition and categorization (see 
Tanaka, 2004, for a review). Since the work of Miller and colleagues (Miller, Freedman, \& Wallis, 2002), it is believed that category learning may be coded in the brain at the single-cell level. For example, individual neurons in the dorsolateral prefrontal cortex in awake, behaving monkeys trained to categorize images of cats and dogs became selective for one or the other category (Freedman, Riesenhuber, Poggio, \& Miller, 2001). Moreover, the possibility that within-basic-level category experience perceptually tunes infants in to features that will be relevant for distinguishing among subordinate-level categories from that basic-level category may also have a neural analogue at the single-cell level, as reported by Sigala and Logothetis (2002). As a consequence of categorization training designed to teach monkeys to differentiate between classes of outline face or fish stimuli, individual neurons in the inferotemporal cortex of the monkeys learned to respond preferentially to features that were diagnostic of the category contrast, relative to features that were present in the stimuli, but not categorically distinguishing. ${ }^{1}$ The operation of such "expert neurons" suggests a neural correlate of expertise at the single-cell level in a part of the brain that has been associated with object recognition (Hasegawa \& Miyashita, 2002). Recent event-related potential (ERP) studies with human adults have shown that subordinate-level categorizations elicit an enhanced negative deflection approximately $250 \mathrm{msec}$ after stimulus presentation, relative to basic-level categorizations (Scott, Tanaka, Sheinberg, \& Curran, 2006; Tanaka, Curran, Porterfield, \& Collins, 2006). ERP studies with infants also indicate that broad global category representations produce slow wave activity in response to stimulus presentation some $200 \mathrm{msec}$ before narrower category representations at the basic level (Quinn, Westerlund, \& Nelson, 2006). The adult and infant ERP studies suggest that brain activity is influenced not only by the amount of category exposure, but also by the level of category exposure.

A further comment concerns the relation between the present work and previously reported findings on plasticity in face processing that have been reported by Pascalis, Nelson, and collaborators (Pascalis, de Haan, \& Nelson, 2002; Pascalis et al., 2005). In the earlier work, 6-month-old infants were shown to discriminate among human faces as well as between monkey faces, whereas 9-month-old infants were shown to discriminate only among human faces. The ability to discriminate among monkey faces was apparently lost, presumably due to lack of experience with monkey faces. However, if the infants were provided with in-home exposure to images of monkey faces in the period between 6 and 9 months, the ability to discriminate among the monkey faces was maintained. Whereas these results indicate that in-home experience within a category of faces can prevent subordinate-level discrimination among faces from within that category from being lost, the findings reported here indicate that laboratory experience within a basic-level category of animals can induce infants to make subordinate-level category distinctions that they otherwise would not. In the same vein, Sangrigoli and de Schonen (2004) have reported that Caucasian 3-month-old infants were able to discriminate between individual adult Caucasian faces, but not between adult Asian faces; however, when the infants were presented with 3 faces (as opposed to just 1) during familiarization, they were able to discriminate among the Asian faces. Both the Sangrigoli and de Schonen results and those reported here suggest that experience that is limited to between 3 and 12 exemplars from a single class can produce rapid perceptual learning that results in the ability to engage in subordinate-level object recognition.

Two final issues raised by the data need to be considered. First, with regard to terminology, although the expertise investigation conducted with adults was discussed in terms of a "training" study (Tanaka et al., 2005), we have chosen not to use this term in the discussion of the present findings. In studies of perceptual expertise with adults, learning can take place over several sessions or even days. It is possible that the familiarization provided here may be more akin to altering the sensory context than to evoking a more permanent learning process. The results may thus be viewed in the framework of an adaptive perceptual filtering rather than a more fixed learning process. An account of this nature emphasizes the recent history of what has been experienced, while still allowing infants the flexibility to learn from among numerous subordinatelevel categories during the course of development.

A second final issue concerns the precise mechanism by which the Part 1 category-learning experience influenced category learning in Part 2. As noted earlier, participants had more exposure to the target basic level in the same basic-level condition than in the different basiclevel condition. That is, while participants were exposed to one or two subordinate-level categories from the same basic-level category, they were also exposed to more (24) or fewer (12) instances from the same basic level. This feature of the experimental design leaves open the question of whether it is multiple subordinate categories or the continued experience within the same basic level that is responsible for the facilitation of category learning in Part 2 of the procedure. Our view is that either possibility is interesting and that additional experimental work will be needed to provide a definitive answer.

\section{Conclusions}

As is the case for adults (Tanaka et al., 2005), experience within a basic-level category is crucial for the creation of exclusive subordinate-level categories. Such experience in infants may tune attention to the surface properties of the exemplars that are diagnostic for subordinate-level contrasts within a given basic-level category. The data suggest that the experiences that promote the acquisition of perceptual expertise in adults may also facilitate the development of perceptual expertise in infants, and imply more generally that the mechanisms that produce perceptual expertise may be operative from the earliest beginnings of development.

\section{AUTHOR NOTE}

This research was supported by NIH Grants HD-42451 and HD46526. Additional research support was provided by grants from the James S. McDonnell Foundation (Perceptual Expertise Network) and 
the National Science and Engineering Research Council of Canada. The authors thank Laura Carlson, Safa Zaki, and two anonymous reviewers for comments on the manuscript, and Laura Hutchins, Lauren Lofdahl, and Laurie Yarzab for assistance in testing participants. Correspondence concerning this article should be addressed to P. C. Quinn, Department of Psychology, University of Delaware, Newark, DE 19716 (e-mail: pquinn@udel.edu).

\section{REFERENCES}

Ashby, F. G., \& SPIERING, B. J. (2004). The neurobiology of category learning. Behavioral \& Cognitive Neuroscience Reviews, 3, 101-113. BEHL-CHADHA, G. (1996). Basic-level and superordinate-like categorical representations in early infancy. Cognition, 60, 105-141.

Eimas, P. D., \& QuinN, P. C. (1994). Studies on the formation of perceptually based basic-level categories in young infants. Child Development, 65, 903-917.

FAGAN, J. F. (1970). Memory in the infant. Journal of Experimental Child Psychology, 9, 217-226.

Freedman, D. J., Riesenhuber, M., Poggio, T., \& Miller, E. K. (2001). Categorical representation of visual stimuli in the primate prefrontal cortex. Science, 291, 312-316.

French, R. M., Mareschal, D., Mermillod, M., \& Quinn, P. C. (2004). The role of bottom-up processing in perceptual categorization by 3- to 4-month-old infants: Simulations and data. Journal of Experimental Psychology: General, 133, 382-397.

Gauthier, I., \& Nelson, C. A. (2001). The development of face expertise. Current Opinion in Neurobiology, 11, 219-224.

Gauthier, I., \& TARr, M. J. (1997). Becoming a "Greeble" expert: Exploring mechanisms for face recognition. Vision Research, 37, 16731681.

GAUTHIER, I., \& TARR, M. J. (2002). Unraveling mechanisms for expert object recognition: Bridging brain activity and behavior. Journal of Experimental Psychology: Human Perception \& Performance, 28, 431446

Hasegawa, I., \& Miyashita, Y. (2002). Categorizing the world: Expert neurons look into key features. Nature Neuroscience, 5, 90-91.

Johansen, M. K., \& Palmeri, T. J. (2002). Are there representational shifts during category learning? Cognitive Psychology, 45, 482-553.

Kelly, D. J., Liu, S., Ge, L., Quinn, P. C., Slater, A. M., Lee, K., ET AL. (2007). Cross-race preferences for same-race faces extend beyond the African versus Caucasian contrast in 3-month-old infants. Infancy, 11, 87-95.

Kelly, D. J., Quinn, P. C., Slater, A. M., Lee, K., Gibson, A., Smith, M., ET AL. (2005). FAST TRACK Report: Three-month-olds, but not newborns, prefer own-race faces. Developmental Science, $\mathbf{8}$, F31-F36.

Miller, E. K., Freedman, D. J., \& Wallis, J. D. (2002). The prefrontal cortex: Categories, concepts, and cognition. Philosophical Transactions: Biological Sciences, 357, 1123-1136.

Oakes, L. M., Coppage, D. J., \& Dingel, A. (1997). By land and by sea: The role of perceptual similarity in infants' categorization of animals. Developmental Psychology, 33, 396-407.

Palmeri, T. J., Wong, A. C.-N., \& GaUthier, I. (2004). Computational approaches to the development of perceptual expertise. Trends in Cognitive Sciences, 8, 378-386.

Pascalis, O., De HaAn, M., \& Nelson, C. A. (2002). Is face processing species-specific during the first year of life? Science, 296, 13211323.

Pascalis, O., Scott, L. S., Kelly, D. J., Shannon, R. W., Nicholson, E., Coleman, M., \& Nelson, C. A. (2005). Plasticity of face processing in infancy. Proceedings of the National Academy of Sciences, 102, 5297-5300.

QuINN, P. C. (2002). Early categorization: A new synthesis. In U. Goswami (Ed.), Blackwell handbook of childhood cognitive development (pp. 84-101). Oxford: Blackwell.

QuINN, P. C. (2004). Development of subordinate-level categorization in 3- to 7-month-old infants. Child Development, 75, 886-899.

QuinN, P. C. (2005). Young infants' categorization of humans versus nonhuman animals: Roles for knowledge access and perceptual process. In L. Gershkoff-Stowe \& D. Rakison (Eds.), Building object cat- egories in developmental time: 32 nd Carnegie Symposium on Cognition (Vol. 32, pp. 107-130). Mahwah, NJ: Erlbaum.

QuinN, P. C., \& EIMAS, P. D. (1998). Evidence for a global categorical representation for humans by young infants. Journal of Experimental Child Psychology, 69, 151-174.

Quinn, P. C., Eimas, P. D., \& Rosenkrantz, S. L. (1993). Evidence for representations of perceptually similar natural categories by 3 - and 4-month-old infants. Perception, 22, 463-475.

QuinN, P. C., Eimas, P. D., \& TARR, M. J. (2001). Perceptual categorization of cat and dog silhouettes by 3- to 4-month-old infants. Journal of Experimental Child Psychology, 79, 78-94.

Quinn, P. C., \& Johnson, M. H. (2000). Global-before-basic object categorization in connectionist networks and 2-month-old infants. Infancy, 1, 31-46.

QuinN, P. C., \& Schyns, P. G. (2003). What goes up may come down: Perceptual process and knowledge access in the organization of complex visual patterns by young infants. Cognitive Science, 27, 923-935.

Quinn, P. C., Schyns, P. G., \& Goldstone, R. L. (2006). The interplay between perceptual organization and categorization in the representation of complex visual patterns by young infants. Journal of Experimental Child Psychology, 95, 117-127.

QuinN, P. C., Westerlund, A., \& Nelson, C. A. (2006). Neural markers of categorization in 6-month-old infants. Psychological Science, 17, 59-66.

Quinn, P. C., Yahr, J., Kuhn, A., Slater, A. M., \& Pascalis, O. (2002). Representation of the gender of human faces by infants: A preference for female. Perception, 31, 1109-1121.

Robbins, R., \& McKone, E. (2007). No face-like processing for objects-of-expertise in three behavioural tasks. Cognition, 103, 34-79.

Rosch, E., Mervis, C. B., Gray, W. D., Johnson, D. M., \& BoyesBRAEM, P. (1976). Basic objects in natural categories. Cognitive Psychology, 8, 382-439.

SAngrigoli, S., \& De Schonen, S. (2004). Recognition of own-race and other-race faces by three-month old infants. Journal of Child Psychology \& Psychiatry, 45, 1-9.

Schyns, P. G., Goldstone, R. L., \& Thibaut, J. P. (1998). The development of features in object concepts. Behavioral \& Brain Sciences, 21, 1-54.

Scott, L., Tanaka, J. W., Sheinberg, D., \& Curran, T. (2006). A reevaluation of the electrophysiological correlates of expert object processing. Journal of Cognitive Neuroscience, 18, 1453-1465.

Sigala, N., \& Logothetis, N. K. (2002). Visual categorization shapes feature selectivity in the primate temporal cortex. Nature, $\mathbf{4 1 5}, 318$ 320

Smith, J. D., \& Minda, J. P. (1998). Prototypes in the mist: The early epochs of category learning. Journal of Experimental Psychology: Learning, Memory, \& Cognition, 24, 1411-1430.

TANAKA, J. W. (2001). The entry point of face recognition: Evidence for face expertise. Journal of Experimental Psychology: General, 130, 534-543.

TANAKA, J. W. (2004). Object categorization, expertise, and neural plasticity. In M. Gazzaniga (Ed.), The cognitive neurosciences (3rd ed., pp. 877-887). Cambridge, MA: MIT Press.

Tanaka, J. W., Curran, T., Porterfield, A. L., \& Collins, D. (2006). Activation of preexisting and acquired face representations: The N250 event-related potential as an index of face familiarity. Journal of Cognitive Neuroscience, 18, 1488-1497.

Tanaka, J. W., Curran, T., \& Sheinberg, D. L. (2005). The training and transfer of real-world perceptual expertise. Psychological Science, 16, 145-151.

TANAKA, J. W., \& TAYlor, M. (1991). Object categories and expertise: Is the basic level in the eye of the beholder? Cognitive Psychology, 23, 457-482.

Thomas, E., Van Hulle, M. M., \& Vogels, R. (2001). Encoding of categories by noncategory-specific neurons in the inferior temporal cortex. Journal of Cognitive Neuroscience, 13, 190-200.

Vogels, R. (1999). Categorization of complex visual images by rhesus monkeys: Part 2, Single-cell study. European Journal of Neuroscience, 11, 1239-1255.

Younger, B. A. (1985). The segregation of items into categories by tenmonth-old infants. Child Development, 61, 614-620. 
Younger, B. A., \& Fearing, D. D. (1999). Parsing items into separate categories: Developmental change in infant categorization. Child Development, 70, 291-303.

\section{NOTE}

1. It should be acknowledged that the evidence of Sigala and Logothetis (2002) is contradicted by the prior work of Vogels and colleagues (Thomas, Van Hulle, \& Vogels, 2001; Vogels, 1999), who reported null results with respect to the issue of whether the firing rate of single neu- rons in the inferotemporal cortex of monkeys could be influenced by category learning. Ashby and Spiering (2004) have offered a possible reconciliation of the discordant findings by suggesting that neurons of the inferotemporal cortex of monkeys may encode a high-level representation of visual stimuli, which in turn provides direct input to categorylearning processes.

(Manuscript received July 4, 2006;

revision accepted for publication September 25, 2006.) 